\title{
Special issue on meteorological disaster risk analysis and assessment: on basis of grey systems theory
}

\author{
Zaiwu Gong · Jeffrey Yi-Lin Forrest
}

Received: 10 September 2013/Accepted: 10 September 2013/Published online: 19 September 2013

(C) Springer Science+Business Media Dordrecht 2013

Disaster risk analysis and assessment are the main content and foundation of the management of all risks associated with disasters. They constitute the knowledge basis for meteorological disaster prediction, prevention, compensation, and all other works related to disastrous weather conditions. Meteorological disaster risk analysis studies what kinds of weather disasters will occur at a certain particular geographic region within a specific timeframe. At the same time, meteorological information system contains numerous kinds of uncertainties and incompleteness. That is, meteorological information system is made up of largely grey information. In recent years, a large number of researchers tried to utilize different theories of uncertainty to analyse meteorological disasters. Grey systems theory (Liu et al. 2010; Liu and Lin, 2006) is one among such theories on uncertainty. It includes such major components as grey correlation analysis, grey clusters, grey prediction, etc., and represents a novel theory that is developed to deal with situations of uncertainty due to poor quality of information. This special issue combines the analysis of meteorological disasters and risks with grey systems theory to formulate a new approach for the analysis and assessment of meteorological disasters and risks. This new approach not only supplements the traditional methods widely used in the area of research on disaster risks, but also develops new instruments that are effective in dealing with meteorological disasters and risks.

\footnotetext{
Z. Gong ( $($ )

College of Economics and Management, Nanjing University of Information Science and Technology, Ningliu Road 219, Nanjing, Jiangsu Province, People's Republic of China e-mail: zwgong26@163.com

J. Y.-L. Forrest

Department of Mathematics, Pennsylvania State System of Higher Education at Slippery Rock, Slippery Rock, PA 16057, USA

e-mail: Jeffrey.forrest@sru.edu
} 


\section{Our reasons for publishing this special issue}

(1) Meteorological disaster risk analysis and assessment are an integral part of "disaster prevention and management".

Meteorological disaster risk analysis constitutes the main content of disaster risk management of weather-related events and plays the fundamental role for meteorological disaster prediction, prevention, compensation, and other relevant studies. Meteorological disaster risk analysis investigates what kinds of natural disasters will appear in special regions within particular timeframes; it analyses the possibilities and aftermaths of such disasters. Meteorological disaster risk assessment characterizes patterns and magnitudes of natural disasters and proposes scientific means and systematic methods to be used for risk reduction. In particular, with meteorological disaster risk assessment in place, risk assessment and disaster management are organically combined; appropriate management measures are designed to reduce or to avoid unaccepted risks so that consequent losses are reduced to their maximal limits. In other words, meteorological disaster risk analysis and assessment serve to produce tangible economic benefits for the society.

(2) An increasing number of countries and international organizations, such as International Council for Science, ICSU, have placed great emphasis on the study of disaster risk analysis and assessment.

Many developed countries have paid great attention to the integrated risk management since the 1990s. ICSU formally proposed a scientific plan regarding integrated risk management in 2008. The integrated risk analysis, which makes joint use of the strengths of natural sciences, technology, and social sciences, has played an important role in maintaining and improving the involvement government of different levels in risk management.

(3) The conventional models of disaster risk analysis and assessment have experienced difficulties in dealing with the uncertainty and incompleteness widely existing in the available information of meteorological disasters. To this end, the grey disaster theory provides a novel approach to deal with such problems.

Meteorological information system is an open complex giant system. That contains numerous uncertainties, which are partly due to randomness, partly due to fuzziness, and partly due to incompleteness that can be well described by greyness. When the randomness is studied by using probabilistic methods and laws of causation, it is usually difficult for the researcher to evaluate whether or not a presumed probability distribution is reasonable. Additionally, he usually runs into the problem that the available samples of data in the meteorological risk information system are too small and the quality of the available information is too poor to be useful. Therefore, it is theoretically difficult for the researcher to uncover the accurate relationship between the event of interest and its probability of occurrence; and it is also practically difficult for him to assure the exact probability distribution of the risk event without sufficiently large samples of data. Such realistic defects widely existing in meteorological information system could be remedied by employing grey systems theory, which has been an integral part of the science of uncertainty.

Grey systems theory represents a new methodology of investigating small samples of data and the uncertainty caused by the poor quality of information. Its main object is to research uncertain systems with small samples and incomplete information. It focuses on generating, excavating, and abstracting valuable information from the partially known information and eventually describing and effectively monitoring the operational behaviours and the evolutionary laws of the system of concern. This special issue on the meteorological disaster risk analysis using grey systems theory employs such technologies 
as grey incidence analysis, grey clustering methods, and grey prediction mechanism. The meteorological disaster risk analysis that utilizes grey systems theory created a novel grey disaster theory, which not only realizes the integration of traditional disaster risk analysis with grey systems methods, but also develops a new instrument for meteorological disaster risk analysis and assessment.

In this special issue, fourteen papers are accepted as full papers. Some of the issues addressed in the fourteen papers include:

\section{Grey correlation analysis}

Grey correlation theory is one of the important contents of grey system theory. The general idea of grey correlation theory is to measure the correlational degree among different discrete sequences based on their geometric similarity or dissimilarity degree of development forms. According to the existing literature, most scholars only subjectively set and calculated the corresponding disaster hierarchy values by paying attention to meteorological disasters in a certain period or region and made disaster loss assessment evaluation of disaster losses based on relative disaster cases. The paper by Y. Zhao, Z. Gong et al. introduces the grey correlation method of risk evaluation in meteorological disaster losses based on historical disaster data in China (mainland) and applies the improved grey relational analysis model (the triangular grey relational model) to the risk evaluation of rainstorm and flood disaster losses. This paper comprehensively considers historical disaster statistics of China's 31 provinces (mainland), selects four indicators (disaster area, disaster population, collapsed houses, and direct economic losses) as dynamic risk evaluation system of disaster loss, and finally constructs China's zoning map of the risk of rainstorm and flood disaster losses, according to grade standard of rainstorm and flood disaster in different regions. The grey correlation method provides reasonable and effective references for national disaster preventions which can be used in other researches focused on risk evaluation of meteorological disaster losses. Grey correlation analysis also provides an important tool to study karst hydrological processes. The paper by $\mathrm{Y}$. Hao, X. Chen, and X. Wang proposes a grey auto-incidence analysis which can identify the periodicity of a time series, for example, precipitation and spring discharge. They apply the method to Liulin Springs Basin, China, and obtain the periodicity of precipitation and spring discharge, respectively. This work reveals that the periodicity of precipitation is 8 years and of spring discharge is 3 years. The difference between the periodicities of precipitation and spring discharge reflects the role of karst aquifer in hydrological processes.

\section{Grey clustering methods}

This special issue presents two kinds of clustering methods: one is grey fixed-weighted cluster method and the other grey relational clustering. The grey fixed weight cluster theory is one of effective methods to assess rainstorm and flood disaster losses, and the method could be commonly used in the assessment of meteorological disasters. Based on the large set of 1,302 historical sample data of the past 6 years from 2004 to 2009 in China mainland, M. Shao, Z. Gong and X. Xu analyse the impact of rainstorm and flood disasters using the methodology of grey fixed weight cluster analysis according to disaster losses, which are divided into the three grey classes of high, medium, and low. The regional differences of the risk assessment of the rainstorm and flood disasters are discussed, and the dynamical risk zoning map is conducted. The results show a consistent conclusion with the actual losses of rainstorm and flood disasters over each administrative district, which can provide more scientific evidence for the relevant governmental departments of disaster 
prevention and mitigation. In coastal areas of China, tropical cyclone disaster losses are directly affected by tropical cyclone risk source. The paper by L. J. Zhang, Z. H, Yun, and $X$. J. Sun uses the grey relational density-based spatial clustering of applications with noise (GR-DBSCAN) analysis of the impact on China's tropical cyclones source distribution. Taking into account climate change, they divide 1949-2008 into three time periods and analyse the number and intensity of tropical cyclones in different time period and different generated sea areas. The paper by N. Xie, J. Xin, and S. Liu focuses on analysing and evaluating the losses of different meteorological disasters of different regions in China. Considering the complex weather features of China, they divide meteorological disasters into drought, floods, tropical storms, snowstorms, and hail disasters. And they construct a novel regional meteorological disaster loss evaluation method based on grey cluster model and give out the detailed steps of the method. Then, the method is used to evaluate the regional meteorological disaster losses. The paper summarizes the historic loss of meteorological disasters, which is helpful for preparing disaster preventions, for evaluating regional meteorological disaster grades and distribution characteristics for better allocation of resources, and for constructing novel meteorological disaster evaluation models.

\section{Grey econometric analysis}

The grey econometric combined model can eliminate the influence of data's random fluctuation or error on model evaluation. Therefore, the model can be used in either the situation with a known system structure or the situation of an uncertain system structure. When studying the relationship between meteorological factors and economic data, most scholars only consider the meteorological data themselves without taking into account the special changes of meteorological data. In addition, scholars rarely consider the influence of disturbance in primitive series in their research. So there always is such a problem that the accuracy of the model is not high enough. Many studies are more about the relationship between meteorological factors and economic data, while only a few about catastrophic data and economic data. The paper by C. Guo, X. Xu, and Z. Gong studies the relationship between the GDP growth rate of Nanjing city (China) and meteorological catastrophic factors. Firstly, they unite root tests on each series and then take co-integration analysis on the stationary series and then they build corresponding error correction models to analyse the long-term and short-term effects of meteorological catastrophic factors on GDP growth rate. Finally, they verify this relationship with Granger causality analysis. The paper by W. Sun, L. Li, and Z. Gong focuses on the relationship among meteorological disaster factors, environmental regulations, and the economic output of manufacturing in Jiangsu, China. Applying the production function and the grey correlation method, this paper analyses the influence of meteorological disaster factors and environmental regulations on the economic output of manufacturing in Jiangsu. By expanding the Cobb-Douglas production function, the meteorological disaster factors and environmental regulations are considered as new inputs which relate to the output of manufacturing together with traditional inputs like capital and labour. The empirical analysis shows that the development of typical manufacturing in Jiangsu, China, is influenced by meteorological and environmental factors.

\section{Grey decision analysis}

In current meteorological disasters evaluation system, there are two major problems that need to be considered. On the one hand, indicators' data are difficult to collect and the information of evaluation indices is very limited. On the other hand, natural disasters have significant spatial and temporal variations, while the comprehensive quantitative 
evaluation criteria of the national meteorological disasters are far from perfect. Since grey systems theory is specific to problems with uncertainty of fewer data and poor information, the paper by Y. He and Z. Gong introduces the grey dynamic decision-making model into the evaluation for meteorological disasters. According to the historical disasters data that come from Yearbook of Meteorological Disasters in China (2005-2010), they quantitatively evaluate the disaster losses of torrential rains and floods in Chinese mainland. Considering the different rainstorm flood grades in different regions, they also construct the national flood disaster situation maps. The results demonstrate that the evaluation method of rainstorm flood disasters is practical and effective. The paper by Z. Gong, Y. Zhao, and X. Ge introduces the data envelopment analysis (DEA) method to analyse the input-output efficiency of energy consumption and economic indicators in Beijing city under the influence of short-term climatic factors. In this paper, with total energy consumption, fixed asset investment, average temperature, precipitation, sunshine hours, average wind velocity and the average pressure being employed as the input variables, and GDP and per capita disposable income being employed as the output variables, they establish DEA model based on the time series data in Beijing city from 1980 to 2010 and analyse the input-output efficiency of DEA of 31 decision-making units under the influence of the short-term climatic factors. Generally, it is confirmed that energy consumption and economic growth conditions have strong sensitivity to the short-term climate factors.

\section{Grey prediction theory}

Grey systems theory provides a modelling and prediction method for time series data with a small sample set. The mechanism is presented as follows: the potential rule behind messy data is generated and excavated through the appropriate series. So far, grey prediction models and methods have already been widely used in economic growth, new industry, company earnings, financial risk prediction, and so on. The grey systems theory has been widely applied to system simulation researches whose features are of small sample and poor information. However, a series whose time spans long will lead to the problem of remarkable fluctuations, which will cause the difficulty of making accurate predictions. Grey prediction theory can effectively smooth such data series, decrease the fluctuation of data series sample, and eventually improve the robust of data series sample. Based on the newly constructed low-temperature index, the paper by Z. Gong and C. Chen and $\mathrm{X}$. Ge smoothes the fluctuating series of low temperatures by employing grey prediction theory. Additionally, it is also shown that Markov prediction model has the obvious advantage on state prediction. In this research, they calculate the days when the temperature is lower than or equal to $-5{ }^{\circ} \mathrm{C}$ in a year according to the data of daily minimum temperature in Nanjing city from 1951 to 2011, divide these days into 6 categories according to the number (one, two, three, four, five, six, and above) of consecutive lowtemperature days, and employ the annual low-temperature weighted index. They verify the effectiveness of weighted Markov model and grey weighted Markov model according to these indexes from 1951 to 2011. Mining subsidence destroys environment seriously and is difficult to forecast because the parameters in prediction model are difficult to obtain. As there are many uncertainties in mining subsidence, H. Xu, B. Liu, and Z. Fang construct a grey model based on matrix sequence of cross-sectional data and forecast land subsidence in coal mine. In this article, they study the geometric meaning of panel data, using projection transformation and volume conversion, and transform cross-sectional data sequence into matrix sequence which includes the same information. They set up a prediction model of matrix sequence. Using the newly created model, they predict the land subsidence of coal mine in Pingdingshan and compare the predicted value with actual value. The results 
show that the prediction effect is good. Thus, the scope of grey prediction has been expanded, and grey prediction theory has been enriched.

6. Other theories on meteorological disaster risk analysis

The paper by L. P. Li, K. M. Zhang et al. investigates the multi-year average characteristics and the inter-annual variation of drought and flood hazards during the pre-rainy season in South China, reveals the causes of the inter-annual anomalies, and analyses the drought and flood hazard risks. Based on the measured rainfall data in Shanghai, Y. Jing, J. Li et al. calculate the drought conditions before the typhoon Saomai in the study area. Moderate-resolution Imaging Spectroradiometer (MODIS) data are used to evaluate the changes of vegetation index and vegetation supply water index as affected by the typhoon. The result shows that the typhoon Saomai could effectively alleviate drought conditions. It has some other advantages such as the decreasing of the summer high temperature except for disadvantages. MODIS remote sensing data combined with measured ground data are a useful quantitative assessment tool in monitoring drought relief by local typhoons. M. Mirzaei, Y. Huang et al. study the extreme rainfalls in the Zayanderood River Basin in central Iran for durations between $24 \mathrm{~h}$ and $120 \mathrm{~h}$, estimate the generalized extreme value (GEV) parameters of this time series using the method of L-moments, and obtain standard deviations among the estimated GEV parameters using the bootstrap method.

Acknowledgments We would like to express our gratitude to Prof. T.S. Murty, Editor-in-Chief of Natural Hazards, for the opportunity to serve as the guest editors for this Special Issue. We would also like to thank all reviewers for providing in-depth comments and constructive criticisms, and the authors for contributing their high-quality manuscripts.

\section{References}

Liu SF, Lin Y (2006) Grey information theory and practical applications. Springer, London Liu SF, Dang YG, Fang ZG (2010) Theories and application of grey system. Science Press, Beijing 